bulgaricus conservées à des températures différentes. Journal of Dairy Science, 1931.

HARRIMAN et HAMmer. Variation de la coagulation et de la protéolyse du lait par le Streptococcus lactis. Journal of Dairy Science, 1931.

BAumann. A propos de la relation entre la teneur en matières sales et le nombre de germes du lait. Pelweizorischen milch., 1932.

Robert S. Breed. Les bactéries thermophiles du lait pasteurisé par la pasteurisation basse. Le Lait, 1933.

Aubel et Srmon. Sur la fermentation lactique, C. R. Soc. Biol., 1933.

Genevois et Nioolaiefr. Potentiel d'oxydo-réduction de la fermentation lactique bactérienne. C. R. Soc. Biol., 1934.

C. GoRIni. La signification des bactéries thermorésistantes et thermophiles dans le lait stérilisé, pasteurisé et cru. Congrès mondial de Laiterie, Rome, Milan, 1934.

Albert Fournier. Sur un coefficient de propreté du lait. "Ligue du Lait", 28 mars 1935. Revue de Path. comp., juin-juillet 1935. - Presse Méd., 21 septembre.

Albert Fournier. Recherches sur l'acidogénie du lait. Bulletin de la Société de Chimie biologique, février 1938.

\title{
PROGRÈS RÉCENTS EN CHIMIE LAITIÈRE
}

par

W. L. DAVIES

(fin)

\section{LES PRODUITS DE L'INDUSTRIE LAITIÈRE}

\section{a) Le beurre}

1. Texture. - Lyons [134] a pu relier la dureté de différents beurres à leur indice d'iode et à leur teneur en acide stéarique. Il a constaté que le beurre de Nouvelle-Zélande et d'Australie était plus dur que celui d'Irlande ou de Danemark et que la non saturation des matières grasses était plus faible dans les beurres les plus durs. L'indice d'iode du beurre est plus élevé lorsque les vaches sont alimentées dans les pâturages : cette valeur augmente au fur et ¿ mesure que la saison avance. La fertilité du sol n'a pas d'influence sur la composition du beurre. Les modifications de la dureté du beurre qui se produisent au début et à la fin de la période de pâturages ne sont pas entièrement dues à une différence de composition, mais aussi à une différence d'état physique des matières grasses qui est elle-même due aux facilités plus ou moins grandes de refroidissement. Coulter et Coombs [135] signalent que l'on améliore la texture du beurre par un refroidissement rapide de la crème à des températures inférieures à $5 \mathrm{C}^{\circ}$. et par un barattage à température aussi basse que possible. 
Richardson et Aввоtт [136] ont observé que les vaches alimentées avec du foin de luzerne donnent un beurre dont la consistance est défectueuse par suite d'une composition anormale des matières grasses. L'aspect collant et émiettable de ce beurre est dû au fait que, pendant que l'on conserve la erème dans les conditions habituelles, la quantité de matières grasses qui cristallise avant barattage est beaucoup plus grande que d'habitude, et il ne reste qu'une trop faible proportion de matières grasses liquides pour donner au beurre de la cohésion, de la plasticité. Le refroidissement rapide de la crème et le barattage immédiat donnent un beurre qui possède une meilleure structure. HoRN [137] signale que l'on améliore la consistance du beurre en incorporant des matières conservées en silo dans la ration habituelle des vaches.

Platon et ses collaborateurs [138] ont constaté que l'on communique au beurre une saveur de poisson lorsqu'on incorpore dans la ration alimentaire des animaux, certains légumes verts, et des betteraves sucrières, mais que l'on risque moins de souiller le beurre en utilisant pour l'alimentation des vaches, de la pulpe de betterave, des graines de céréales, et du foin. La variation de l'indice d'iode en fonction de la nature de la ration alimentaire est la suivante: luzerne seule, 45 à 47 ; trèfle seul, 46 ; mélange de 70 parties de luzerne et de 30 parties de céréales, 39 ; betteraves $(75 \%), 29$; trèfle $(40 \%), 36$.

2. Odeur et saveur. - On a étudié avec un soin tout particulier le développement de l'arôme dans la crème et dans le beurre. On a perfectionné les méthodes permettant de rechercher et de doser le diacétyle. On a aussi recherché le mécanisme de la production du diacétyle. Hammer [139] considère que la saveur du beurre provient de deux origines principales : la matière grasse elle-même, et les produits métaboliques des organismes qui vivent dans la crème. Il attire l'attention sur la formation simultanée du diacétyle et de l'acétone au cours de la maturation de la crème : il montre que l'acétone se transforme lentement en diacétyle, que le beurre contient en moyenne 2 à 4 p. p. m. de diacétyle, et qu'une teneur élevée en diacétyle correspond à une faible qualité de conservation du beurre.

On a utilisé la réaction de Voges.Proskauer pour la recherche du diacétyle et de l'acétone, afin de déterminer la teneur de la crème et du beurre en substances génératrices de saveur. La vitesse de développement et la profondeur de la teinte obtenue en mélangeant de la soude concentrée et une petite quantité d'une culture contenant de la créatine, varient comme l'intensité de la saveur communiquée au beurre $[140,141]$.

BARNICOAT [142] a amélioré et standardisé les méthodes de 
dosage du diacétyle et de l'ensemble (diacétyle + acétone). Le diacétyle est entraîné à la vapeur dans une atmosphère de gaz carbonique, et l'ensemble (diacétyle + acétone) est entraîné à la vapeur après oxydation de l'acétone en diacétyle par une solution de chlorure ferrique; on dose l'ensemble à l'état de diacétyle. On fixe le diacétyle par de l'hydroxylamine. Le composé de nickel et de la diméthylglyoxine formée est ensuite préparé, extrait avec du chloroforme et dosé colorimétriquement par comparaison avec des solutions contenant des quantités déterminées du complexe de nickel. L'auteur conseille d'amener à sec la solution dans laquelle on a formé le complexe de nickel, car l'extraction de ce produit par le ehloroforme est difficile lorsque l'on est en présence d'eau. Kunze [143] propose une méthode de microdosage gravimétrique dans laquelle on pèse le précipité du complexe de nickel.

Les études biochimiques relatives au développement de l'arôme ont principalement porté sur l'influence de l'acidité et de la teneur en oxygène des cultures, sur la quantité de diacétyle formé et sur le rapport : diacétyle-acétone. Michaelian et Hammer [144] ont étudié l'oxydation de l'acétone en diacétyle dans le beurre. Ils ont constaté qu'en ajoutant de l'acétone dans du lait stérilisé et en y faisant barbotter du gaz renfermant de l'oxygène on ne pouvait pas réussir à former de quantités appréciables de diacétyle. Ils en concluent que c'est l'activité des organismes qui est le principal facteur de l'oxydation de l'acétone; l'oxygène est toutefois nécessaire pour accroître la quantité de diacétyle formée par les organismes. Virtanen et ses collaborateurs [145] indiquent que l'oxygène est nécessaire non seulement pour cette oxydation mais aussi pour accroître la quantité d'acétone formée. Certains organismes qui ne produisent que peu d'acétone en eouches épaisses, en donnent des quantités importantes en couches minces. RITTER et CHRISTEN [146] ont constaté que l'addition de citrates a pour conséquences l'augmentation de la quantité d'acides volatils de diacétyle et d'acétone dans les cultures de beurre; la quantité de matières fournies dépend de la durée de l'incubation. La proportion d'acides volatils et d'acétone peut varier dans d'assez grandes limites sans que la saveur du beurre soit beaucoup modifiée.

HAMMER et ses collaborateurs [147] ont montré que lorsqu'on ajoute du diacétyle et de l'acétone à un milieu dans lequel des organismes d'acide lactique sont en pleine croissance, ces corps sont partiellement réduits à l'état de 2-3-butylène glycol; cette transformation s'effectue à un $p \mathrm{H}$ compris entre 3,8 et 4 . La même transformation s'effectue dans les cultures de lait à forte et à faible acidité. L'addition d'acide eitrique dans du lait non stérilisé aug. mente la vitesse de formation du diacétyle et de l'acétone. La neu. 
tralisation des cultures facilite la disparition de l'acétone, tandis que l'eau oxygénée et une température inférieure à zéro degré retardent cette disparition. Les expériences nous renseignent doncsur ce qui se passe dans l'industrie laitière. La vitesse de réduction de l'acétone en glycol est plus grande dans le beurre neutralisé que dans le beurre non neutralisé. La réduction est également assez rapide dans une solution à $12 \%$ de chlorure de sodium. VirTanen et TARNANEN [148] indiquent que le diacétyle n'est pas formé par l'interréaction de deux molécules, ce qui donnerait du diacétyle et du butylène glyeol, mais seulement lorsque l'oxygène entre en relation et s'empare d'atomes d'hydrogène. Ils considèrent que la plus grande partie du diacétyle est formée pendant la préparation du beurre car la crème est mûrie dans des conditions d'anaérobiose et est ensuite barattée en présence d'autant d'air que possible. La perte de l'arôme est due à la présence de bactéries dans le beurre.

BARNICOAT [149] a trouvé de l'acétone et du diacétyle dans du beurre de Nouvelle-Zélande fabriqué à partir de crème légèrement mûrie. Lorsque le beurre ne contient que de faibles quantités de ces produits, on ne constate pas de grandes modifications après une conservation de six mois, mais dans les beurres qui en contiennent 4 pour mille on peut voir d'importantes transformations.

RUEHE [150] suggère de régler la saveur du beurre par addition de distillats de cultures. Il conseille d'ajouter du citrate à des cultures âgées de 24 heures, de laisser incuber pendant une nouvelle période de 24 heures, d'ajouter du chlorure ferrique, puis de distiller le diacétyle et de former ainsi une solution aqueuse. On standardise cette solution de diacétyle et on en ajoute au beurre 5 pour mille, au moment où l'on effectue le salage. Le beurre ainsi préparé se conserve parfaitement bien.

Lemoigne et MonguiLlon [151] sont également partisans d'améliorer la saveur du beurre par addition de diacétyle provenant d'une source naturelle, mais refusent d'admettre que des distillats. eontenant 5 à 7 milligrammes de diacétyle par litre soient des. produits naturels.

RIDDET et ses collaborateurs [152] ont recherché quelles sont les sortes de plantes qui donnent au beurre et à la crème leur saveur. Ce sont principalement les trèfles blanes et rouges; les herbes luxuriantes n'ont pas d'influence. L'herbe printanière fraíche donne au beurre un goût de coumarine. L'emploi d'engrais favorise la pousse de l'herbe et contribue indirectement à l'amélioration de la saveur du beurre.

Ritter [153] indique que le goût de poisson de certains beurres est dû à la triméthylamine, formée à partir de la bétaine ou à partir 
d'une portion de la lécithine par une action hydrolytique oxydante catalysée par des traces de métaux lourds ; on peut régler la saveur du beurre par un choix judicieux des matières alimentaires consommées par les vaches, par l'utilisation de crème fraîche et par pasteurisation de celles-ci à $90^{\circ} \mathrm{C}$.

3. Qualité de conservation. - Les facteurs qui agissent sur les qualités de conservation du beurre ont été décrits par OverMAN [154]. Le beurre fabriqué à partir de crème fraîche se conserve mieux que celui qui est obtenu à partir de crème mûrie. Le beurre fabriqué à partir de crème neutralisée après maturation par ensemencement est meilleur que celui que l'on obtient après neutralisation de crème munie sans aucun ensemencement. La nature du produit utilisé pour effectuer la neutralisation n'a pas d'influence sur la conservation, mais si l'on a dépassé le point de neutralité on obtient un beurre qui se conserve mal. Les meilleurs beurres sont obtenus par barattage du lait frais entier : après deux ans de conservation, les beurres ainsi fabriqués étaient encore vendables.

BARNICOAT [155] a constaté que le beurre enveloppé dans des boîtes doublées d'étain, ou dans du parchemin recouvert de feuilles d'aluminium, se conserve mieux à $-10^{\circ} \mathrm{C}$. qu'à zéro. Le meilleur mode de conservation est l'empaquetage en boittes doublées d'étain et le maintien d'une température de $-20^{\circ} \mathrm{C}$. Le beurre doit être enfermé dans cette atmosphère froide, aussitôt que possible. Hood [156] a remarqué au Canada que l'on peut réduire la souillure due au bois, par un traitement préalable de l'intérieur des boîtes avec de la caséine-formaldéhyde. KIEFERLE et SENSS [157] ont classé les différents modes d'empaquetage suivant un ordre d'efficacité croissante : aucune enveloppe, parchemin, cellophane "ultrament", feuilles d'étain ou d'aluminium. $L$ ' "ultrament» est un parchemin. végétal spécial imprégné d'un colorant végétal jaune.

Gilmour et Arup [158] affirment que le réglage et la surveillance de l'acidité à l'aide de mesure de $p H$ permettent de déterminer les qualités de conservation d'un beurre. Les beurres qui ont un $p$ H inférieur à 6,7 se conservent mal. Les beurres qui possèdent une saveur forte et un $p H$ élevé se conservent bien, mais ceux qui ont une faible saveur et un $p H$ élevé se conservent mieux encore.

GUTHRIE et ses collaborateurs [159] montrent que la pasteurisation à $74^{\circ} \mathrm{C}$. détruit toutes les enzymes nuisibles, mais que si l'on opère à $64^{\circ} \mathrm{C}$. pendant 30 minutes, toutes les enzymes ne sont pas détruites. Les enzymes peuvent exister en présence de sel, mais non pas dans un milieu dont le $p$ H est inférieur à 4,7. JACOBSEN [160] a observé que les beurres qui contiennent les plus fortes teneurs en azote aminé sont ceux qui ont les indices les plus bas; il conseille d'effectuer le dosage de l'azote qui existe sous cette forme pour 
compléter la série des essais relatifs à la saveur et à l'acidité du beurre. KNUDSEN [161] a trouvé une relation entre la qualité et la teneur de 16.000 échantillons de beurre en catalase : le coefficient $(\mathrm{z})$ est compris entre $-0,85$ et $-0,96 \%$.

4. Divers. - Trambics et Pasztor [162] constatent que le beurre qui contient de 1,2 à 2,4 pour mille de cuivre prend rapidement une saveur huileuse. Les beurres commerciaux contiennent de 0 à 4,5 pour mille de fer, et de 0 à 2,0 pour mille de cuivre.

French [163] décrit une méthode de préparation d'un beurre clarifié ayant d'excellentes qualités de conservation, à partir de crème mûrie pendant un temps assez court (12 à 18 heures). Le beurre doit être lavé à fond et clarifié aussitôt à la température d'ébullition.

On peut doser les impuretés contenues dans le beurre en traitant 100 grammes de beurre par $200 \mathrm{~cm}^{3}$ d'une solution à $4 \%$ de borax. On fait bouillir et on filtre dans un entonnoir de Buchner. On lave le filtre avec du pétrole [164].

\section{b) Fromage}

1. Emploi de la présure et fabrication. - Powell [165] a confirmé les résultats obtenus par divers auteurs : La pasteurisation du lait à $63^{\circ} \mathrm{C}$. pendant 30 minutes suivie d'un refroidissement à $35^{\circ} \mathrm{C}$. et de l'addition immédiate de présure, ou le vieillissement à $5^{\circ}, 35^{\circ}$ ou $65^{\circ} \mathrm{C}$. pendant 3 heures n'ont que peu d'influence sur la rapidité de coagulation. La pasteurisation rapide à $75^{\circ} \mathrm{C}$. n'a également qu'une faible influence, mais un chauffage plus prolongé à une température plus élevée augmente la durée de coagulation.

Koestuer et MoDowall ont poursuivi leurs recherches relatives à la fabrication du fromage. Koestrer et Petermann [166] ont examiné les facteurs qui peuvent empêcher la séparation du petitlait et du caillot. Ils n'ont pas trouvé de relations directes entre la cause de la coagulation et la vitesse de séparation du petit-lait. Il n'y a pas davantage de relation entre la vitesse de coagulation et le volume de petit-lait. La séparation du petit-lait est d'autant plus rapide que le lait est plus acide. C'est une propriété du gel de paracaséinate, de prendre une structure rigide qui règle la séparation du petit-lait. Cette séparation est arrêtée par un refroidissement à $25^{\circ} \mathrm{C}$. Elle est maximum à $45^{\circ} \mathrm{C}$. Un lait difficile à coaguler donne un caillot qui rejette lentement son petit-lait, une quantité importante de matières grasses est entraînée. Si l'on ajoute ce lait dans du lait normal on augmente la quantité de matières grasses contenues dans le petit-lait, on retarde le moment où il commence à se séparer mais on ne modifie pas le volume total du petit-lait obtenu. Le caillot obtenu à partir du lait écrémé ne rejette pas son petit-lait 
à une vitesse plus grande que celui que l'on obtient à partir du lait entier; la teneur en caséine n'a pas d'influence.

DoLby et ses collaborateurs [167] continuant leurs recherches relatives à la fabrication du fromage Cheddar ont constaté que l'acidité $d u$ petit-lait a une influence assez importante sur la teneur du fromage en matières minérales et sur sa texture. L'acidité du fromage dépend de l'acidité du petit-lait et de la quantité de lactose qui reste dans le caillot. L'addition de lactose dans le caillot donne un fromage plus acide mais qui contient encore une certaine quantité de lactose non transformé après 4 mois. Dans le petit-lait on a pu constater qu'il existe une relation étroite entre le $p \mathrm{H}$ et la concentration de l'acide lactique; les variations de la teneur du petit-lait en matières minérales, qui sont dues à des différences d'acidité, ont une légère influence. Il en est particulièrement. ainsi lorsque le petit-lait s'échappe lentement.

McDowall et Dolby [168] ont constaté que le sel ne pénètre pas rapidement à l'intérieur des particules du caillot, mais que la teneur en sel est uniforme après 12 heures. La fermentation du lactose dans le lait caillé, et sa transformation en acide lactique sont quantitatives. Les auteurs mettent en évidence que le caillot renferme une quantité d'eau fixée qui atteint 0 gr. 2 à 0 gr. 3 par gramme de lait caillé sec, et que la distribution des électrolytes entre le caillot et l'eau est régie par une loi d'équilibre.

KoESTLER [169] a remarqué que l'Emmental frais cède de l'eau à des solutions concentrées de sel, mais que le fromage vieux n'a pas cette propriété. Il considère que ce phénomène est un indice du pouvoir absorbant d'eau que possède le lait caillé. Dans ce fromage le pouvoir de gonflement atteint son maximum au moment de la formation des trous et on obtient des variétés de fromage différentes lorsqu'on utilise différentes espèces de lait eaillé n'ayant pas le même pouvoir gonflant. La déformation élastique de ce fromage est une propriété physique qui dépend non pas de la consistance du fromage, mais des propriétés de la caséine.

L'influence de la tension de coagulation sur la fabrication du fromage a été étudiée par SANDERS [170] qui a constaté que l'essai de Hirl n'est pas satisfaisant pour déterminer la fermeté du caillot. L'essai de présure avec l'appareil de Hill est plus satisfaisant. Il existe une relation entre le $p \mathrm{H}$ du lait, la durée de coagulation et la tension de coagulation. L'homogénéisation, la pasteurisation et la présence de lait provenant de vaches atteintes de mastite diminuent la tension de coagulation; ces deux derniers facteurs accroissent également la durée de coagulation.

On constate que la qualité du fromage fabriqué à partir de lait pasteurisé est plus uniforme [171], mais ce fromage a besoin d'une durée de maturation plus prolongée. 
McDowall [172] a étudié la possibílité de transformer le lait en fromage. Il discute le rapport $\frac{\text { caséine Walker }}{\text { matières grasses }}$ en ce qui concerne la quantité de fromage que l'on peut obtenir par livre de matière grasse. Il propose une formule qui permet de déterminer la quantité de fromage frais que l'on peut obtenir à partir de 100 livres anglaises de lait : $2,18 \mathrm{C}+1,17 \mathrm{~F}$ livres.

C étant la caséine Walker et F, la quantité de matières grasses.

2. Maturation du fromage. - SHERWOod [173] a étudié le rôle joué par la pepsine et la présure dans la maturation du fromage Cheddar. La pepsine produit une rupture de protéines, moins importante que la présure. La nature des combinaisons de l'azote contenu dans le fromage mûr est caractéristique de l'enzyme utilisée. Si l'on augmente la proportion de présure, on augmente la rupture des protéines mais on n'apporte aucun changement à la forme sous laquelle se présente l'azote. Une première indication comparative du pouvoir destructeur des enzymes utilisées peut être obtenue, on méconnaît la dégradation que ces enzymes produisent dans le lait. SHERWOOD indique que les expériences effectuées avec des enzymes préalablement chauffées n'ont pas mis en évidence le mécanisme du rôle joué par ces enzymes au cours de la maturation du fromage.

Grimmer et Lange [174] ont constaté que le caséogluten formé au cours de la maturation du fromage de Tilsitt n'est pas un produit de composition uniforme mais qu'il peut être divisé en trois fractions ayant des propriétés et des compositions différentes. La teneur en caséogluten décroît lentement après dix semaines de maturation. Parisi et Devito [175] ont isolé des polypeptides renfermant du phosphore, résistant à l'hydrolyse par les acides ou les enzymes, mais facilement hydrolysées par les alcalis et la phosphatase.

\section{c) Autres produits de l'industrie laitière}

1. Crème. - England et Mecham [176] ont constaté que la fouettabilité de la crème et la stabilité du produit fouetté dépendent de la dimension des globules de matières grasses. La crème de Guernsey $(16,3 u)$ est à ce point de vue supérieure à celle de Ayrshire $(10,6 \mu)$. L'addition d'un mélange d'alginate de sodium et de gelatine augmente la stabilité de la crème fouettée. La décantation de la crème de café mise en bouteilles ( $20 \%$ de matières grasses) est due à des températures de pasteurisation et de séparation trop basses, à des températures de crémage trop élevées, à une quantité trop forte de sels de calcium solubles, et à l'agitation défectueuse [177]. Cette décantation est réduite en utilisant des températures de 
pasteurisation et de séparation élevée, et de crémage basse. L'acidité du café et de la erème fouettée ( $32 \%$ de matières grasses) doit être de 0,12 à $0,14 \%$. Lorsque la crème est maintenue dans un récipient sous une pression de $6 \mathrm{~kg}$. 3 par centimètre carré avec de l'oxyde nitreux, et que l'on fait sortir cette crème par pression, on obtient aussitôt de la crème fouettée [178].

JACKSON et ses collaborateurs [179] ont examiné la décoloration et la corrosion des récipients en étain dans lesquels on conserve la crème. Les deux principaux défauts sont les suivants : $a$ ) coloration rouge de l'étain due à la formation d'un sulfure stanneux; $b$ ) perce. ment du récipient, et taches noires dans la crème. La coloration pourpre est due à une durée trop prolongée de la stérilisation; l'addition de 1 gramme de bicarbonate de sodium par litre de crème réduit le développement de cette coloration. Les taches noires ne se produisent que si du fer a été mis directement au contact de la crème.

Loveless [180] a étudié la variation de la viscosité de la crème de conserve en fonction du chauffage. L'emploi d'une température de ehauffage préalable de $60^{\circ} \mathrm{C}$. au lieu de $43^{\circ} \mathrm{C}$. augmente la viscosité de $50 \%$ et diminue la tendance de la crème à devenir granuleuse.

2. Crème glacée. - On a décrit l'emploi d'alginate de sodium [181]. La gélatine et l'alginate possédent la même influence sur la durée de fouettage de la crème, mais le produit de l'alginate fond plus rapidement à la température ambiante. On améliore la qualité des crèmes glacées en y incorporant simultanément $0,2 \%$ de peotine et $0,3 \%$ de gélatine [182]. DAHLE [183] a constaté que la lécithine d'œuf diminue la durée du fouettage tandis que la lécithine de soja l'augmente.

LUCAS [184] examine les limites de l'emploi de lait écrémé en poudre, et de levure dans le mélange. Les précautions qu'il faut prendre pour battre la crème d'une façon satisfaisante résident dans le choix de matières premières de bonne qualité et dans l'exécution d'une homogénéisation convenable. Young [185] suggère de surmonter l'influence d'une trop grande quantité de lactose dans les mélanges ayant une forte teneur en matières solides, par du lactase, des caséinates, ou par centrifugation du lactose contenu dans le lait condensé après addition de sucrose.

3. Lait condensé. - Stebnitz et Sommer [186] ont étudié l'influence des sels sur l'épaississement du lait concentré au cours de son vieillissement. On ne peut pas augmenter la stabilité d'un lait condensé stable, par addition de citrate, de phosphate ou d'acétate de ealcium, mais un lait instable peut être stabilisé par 
addition de citrate soit avant le préchauffage soit dans le produit fini. L'addition d'acétate de calcium ou de phosphate de sodium à un lait stable produit un épaississement rapide. Il n'y a aucune relation entre l'essai à l'alcool et la stabilité à épaississement.

4. Petit-lait. - Davies [187] donne les résultats d'analyse de vingt échantillons de petit-lait sec. Un produit moyen contient : matières grasses, 1,27 ; protéines, 12,5 ; cendres, 7,5 ; chlorure de sodium, 2,3; acidité titrable (exprimée en acide lactique), 2,4; lactose, 69,4 ; humidité, $7,0 \%$. On a breveté des méthodes de séchage du petit-lait en bloes [188] ou sur des rouleaux après concentration [189]. Le précipité séparé du petit-lait à $70^{\circ} \mathrm{C}$. $(p \mathbf{H} 4,4$ à 4,8$)$, a été dissout dans une quantité suffisante de bicarbonate de sodium ou d'ammonium, de carbonate ou d'hydrate, de façon à atteindre un $p H$ compris entre 6,4 et 6,7. Aussitôt après dissolution, on évapore à siccité, on obtient ainsi une lactalbumine soluble [190].

\section{ANALYSE DU LAIT ET DES PRODUITS DÉRIVÉS}

1. Echantillonnage. - MEADE et LECKIE [191] ont recherché la concordance que l'on pouvait obtenir entre les dosages de matières grasses dans des échantillons de laits globaux ou de laits frais périodiques, prélevés pendant une période de six mois. On a constaté qu'il y a de grandes différences d'un mois à l'autre, et même d'un jour à l'autre, si l'on considère le lait provenant d'un seul troupeau. Les échantillons de lait frais recueilli périodiquement contiennent $0,09 \%$ de matières grasses de plus que les échantillons de laits globaux. $60 \%$ des échantillons globaux contiennent moins de matières grasses, $12 \%$ en renferment autant et $27 \%$ en contiennent plus que les échantillons frais. TAUSsig [192] a constaté que des essais effectués chaque semaine, chaque quinzaine ou chaque mois, donnent des résultats aussi satisfaisants.

2. Acidité. - En titrant l'acidité du lait avec un alcali, la fin de la réaction indiquée par la phénolphtaléine tombe de 9,1 à 8,4 lorsque la concentration de l'indicateur passe de 0,005 à $0,08 \%$ [193]. Il est nécessaire d'ajouter une quantité d'indicateur suffisante pour atteindre la plus forte concentration, quand on veut mesurer exactement l'acidité d'un lait.

McDowall [194] conseille d'effectuer le dosage avec une solution de soude $\mathrm{N} / 10$ jusqu'à ce que l'on obtienne une coloration identique à celle que donnent quelques gouttes d'une solution à $0,01 \%$ d'acétate de rosaniline dans un même volume de lait.

3. Teneur en matières grasses. - La "Bristish Standards Institution " [195] a décrit et donné les caractéristiques d'un appareil standard et de méthodes de dosage des matières grasses du lait 
et des produits dérivés basés sur l'application des méthodes de Gerber. On trouvera dans cette relation les détails relatifs à la qualité et aux dimensions de l'appareil, aux spécifications des réactifs et des méthodes qu'il faut employer pour le lait entier, le lait écrémé, la crème, le fromage et le lait en poudre. Gouding [196] a constaté qu'il y avait une différence constante de $0,05 \%$ entre les résultats des dosages des matières grasses dans le lait suivant que l'on utilise la méthode d'Alfa-Laval (Lindström) ou celle de Gerber.

Konnig [197] décrit une méthode alcaline permettant la séparation des matières grasses du lait, basée sur l'emploi de soude caustique, de sel de Rochelle, d'ammoniaque et d'un mélange d'alcools. Au moyen d'un dispositif spécial on peut mesurer cette quantité de matières grasses : d'après l'auteur, les résultats de cette méthode ne diffèrent pas de plus de $0,1 \%$ de ceux que l'on obtient par la méthode de Gerber. Leithe [198] indique des méthodes réfractométriques permettant de déterminer la quantité de matières grasses contenues dans le lait et le fromage : il utilise comme solvant le bromonaphtalène. Le dosage des matières grasses du lait dans les produits alimentaires à base de céréales dépend des indices de Reichert-Meissl et de Kirschner, des matières grasses que l'on a pu extraire [199].

4. Protéines. - McDowall et McDowell [200] déterminent la quantité de caséine, après précipitation acide, par un titrage au formol. La caséine contenue dans $20 \mathrm{~cm}^{3}$ de lait, précipitée à son point isoélectrique est lavée et dissoute au bain-marie dans $11 \mathrm{~cm}^{3}$ de solution $\mathrm{N} / 10$ de soude, Après neutralisation jusqu'au virage de la phénolphtaléine, on ajoute $4 \mathrm{~cm}^{3}$ de formaline et on titre le mélange jusqu'au même virage. La teneur en caséine est égale au produit du nombre de centimètres cubes de solution $\mathrm{N} / 10$ de soude, par 1,05.

Leroy [201] détermine la quantité totale de protéine par une rapide méthode colorimétrique dont le degré de précision est de $0,1 \%$ de la quantité des protéines. On ajoute à $10 \mathrm{~cm}^{3}$ de lait neutralisé, de la phénolphtaléine, $1 \mathrm{~cm}^{3}$ de formaldéhyde neutre et $10 \mathrm{~cm}^{3}$ de solution de soude contenant soit (A) 0,625 , soit (B) 0,745 grammes de soude par litre. La solution (A) neutralise le lait contenant exactement 29 grammes de protéines par litre, tandis que la solution (B) neutralise celui qui en contient 35 grammes. On compose la teinte rose des solutions obtenues avec des couleurs étalons et on en déduit la teneur en protéine du lait.

TURNAU [202] conseille de déterminer la quantité d'albumine par la différence de l'indice de réfraction du sérum dont on a extrait les protéines, et du sérum dont on a extrait la caséine. On 
extrait la caséine des matières alimentaires à base de céréales et de lait, par un traitement à $40^{\circ} \mathrm{C}$. avec une solution diluée de soude caustique, suivie d'un lavage avec une solution à $1 \%$ d'oxalate de sodium ; on dose ensuite la protéine par un titrage au formol. Le produit de cette mesure par 0,715 donne le pourcentage de caséine (40 grammes de substance, extrait porté à $500^{\circ} \mathrm{C}$. et emploi de $10 \mathrm{~cm}^{3}$ pour le dosage) [203].

5. Lait provenant de différentes espèces d'animaux. Polonovski et Martin [204] différencient le lait de femme du lait de vache par la méthode suivante : on neutralise $1 \mathrm{~cm}^{3}$ de lait jusqu'à virage de la phénolphtaléine. On ajoute 6 gouttes de méthyl orange et on verse une solution d'acide sulfurique N/10 jusqu'à ce que la couleur change. Il faut verser $2 \mathrm{~cm}^{3} 5$ d'acide si l'on avait du lait de femme, et $10 \mathrm{~cm}^{3}$ pour du lait de vache. La quantité de lait de vache introduite dans le lait de femme peut être déduite d'après la loi des mélanges. Il faut modifier ces chiffres lorsque le lait est aigri. Le lait de femme donne une coloration rouge violet lorsqu'on le chauffe avec de l'ammoniaque. On peut déceler la présence de lait de chèvre dans le lait de vache par une teneur en acide caprylique beaucoup trop élevée dans les matières grasses [205].

6. Fraude, neutralisants et épaississants. - On a cherché une relation entre la densité et la viscosité du lait. La viscosité peut être comprise entre 1,58 et 2,00 . On a établi des tables qui indiquent les valeurs qui correspondent au lait entier et au lait falsifié [206]. Mohler et Forster [207] ont étudié l'influence de l'addition d'eau au lait et à la crème, sur la valeur de nombreuses constantes physiques.

ScHWARTZ et ses collaborateurs [208] utilisant trois méthodes de recherche ont trouvés de l'acide benzoïque dans 22 fromages, sur 135 échantillons examinés. MacLachLAN [209] a constaté qu'il était difficile d'obtenir des résultats précis pour le dosage de la formaldéhyde par la méthode de Herner si l'acide sulfurique contient de l'anhydride sulfureux. Il conseille l'addition d'ions ferriques; l'addition de permanganate à l'acide n'a qu'une influence temporaire.

Les caillots de beurre contenant des borates ne brunissent pas aussitôt lorsque l'on chasse l'humidité à une température supérieure à $100^{\circ}$ C. [210]. Les caillots de lait contenant de la sève de banane prennent une coloration rose lorsqu'on les traite par de l'acide chlorhydrique ( 1 à 5 parties de lait) et que l'on fait bouillir pendant 5 minutes [211].

On a conseillé l'emploi de nouvelles méthodes pour la recherche 
du lait neutralisé. TAPERNoux [212] a observé que si l'on fait bouillir $30 \mathrm{~cm}^{3}$ de lait ordinaire avec $0 \mathrm{~cm}^{3} 25 \mathrm{~d}^{\prime}$ une solution à $30 \%$ de chlorure de calcium, on obtient un sérum clair, mais que si l'on avait ajouté préalablement au lait du carbonate ou du bicarbonate de sodium ou de lithium, on aurait obtenu un sérum trouble. On a recherché également la présence d'un excès d'ions sodium. On fait bouillir pendant 3 minutes du lait auquel on a ajouté 1 goutte de solution à $30 \%$ d'oxalate de potassium par centimètre cube de lait. Lorsqu'on ajoute de la phénolphtaléine, le lait neutralisé prend une coloration rose intense [213]. WiLle [214] dose le sodium à l'état d'uranylacétate de zinc et de sodium et détermine si le lait a eté neutralisé ou non d'après la quantité de sodium trouvé. Il ne tient pas compte de la variation considérable de la teneur en sodium du lait, et n'a pas cherché à calculer cette valeur d'après la teneur en chlorure du lait comme l'ont suggéré JoNES et Davies [215] qui ont trouvé la relation suivante :

$\mathrm{Cl}(\mathrm{mg} \%)=\mathrm{Na}(\mathrm{mg} \%) \times 1,24+18,1$.

On a éprouvé de grandes difficultés à obtenir des résultats dignes de confiance pour le dosage de la gélatine par la méthode de Stokes (acide picrique) dans les produits dérivés du lait lorsque la protéine a été plus ou moins détruite. Richardson [216] a constaté que pour la crème on obtient de bons résultats en ajoutant au filtrat de nitrate mercurique, une solution d'acide trichloracétique. Ferguson et Racicot [217] ont rencontré des difficultés en utilisant une solution de nitrate de plomb avec la crème aigre ou certains fromages domestiques contenant de la présure. Ils n'ont obtenu qu'un résultat faiblement positif pour une teneur de $0,5 \%$ en gélatine. L'acide tannique, au contraire, a donné des résultats positifs même quand le produit ne contenait pas de gélatine. L'acide tannique ne permet pas de distinguer la présure de la gélatine. Les auteurs ont constaté que parmi tous les essais, celui de Stokes était le plus utile.

7. Point de congélation. - StubBs [218] a comparé les appareils de Hortvet et de Monier Williams et constate que dans les deux appareils il y a un transport de chaleur résiduaire et que ee phénomène est plus important dans l'appareil d'Hortvet. L'auteur décrit une nouvelle modification de l'appareil : il laisse entre les deux tubes un espace de $2 \mathrm{~mm}$. 5 dans lequel l'alcool est siphonné lorsqu'on effectue la lecture. Ostermann [219] signale des cas dans lesquels le point de congélation du lait de vaches bien portantes, peut être anormal. On a obtenu comme valeur de $\Delta$ :

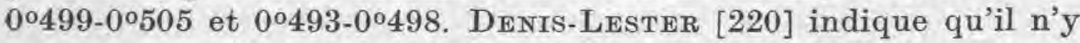
a aucune corrélation entre $\Delta$ et l'époque de l'année. Il a trouvé, 
en examinant le lait de 7 troupeaux, des valeurs de $\Delta$ comprises entre $0^{\circ} 528$ et $0^{\circ} 555$ (moyenne $0^{\circ} 542$ ).

Elsdon et StrubBs [221], en calculant à partir de $\Delta$, la quantié d'eau qui a été ajoutée au lait, tiennent compte de la teneur totale en matières solides. Au lieu de la formule : eau ajoutée $W=100$ $\frac{\left(\mathrm{T}-\mathrm{T}_{1}\right)}{\mathrm{T}}$, ils proposent la formule $\mathrm{W}=\left(\mathrm{T}-\mathrm{T}_{1}\right) \frac{(100-\mathrm{TS})}{\mathrm{T}}$ dans laquelle $\mathrm{T}=\Delta$ moyen $\mathrm{T}_{1}=\Delta$ trouvé et $\mathrm{TS}=$ teneur totale en matières solides.

8. Divers. - En se basant sur la teneur en lactose [222] on a déterminé la quantité de matières sèches du lait contenues dans le pain. Munsey [223] a constaté que les calculs basés sur la teneur en acide citrique ne donnent pas des résultats dans lesquels on puisse avoir confiance. Il extrait toutes les matières grasses et détermine l'indice de Reichert, puis admettant les valeurs de 31,5 pour les matières grasses du lait et 1,0 pour les matières grasses des céréales, il calcule d'après la loi des mélanges la quantité de matières grasses provenant du lait. Afin d'éviter la perte d'acide lactique par volatilisation [224] au cours de la détermination des matières solides contenues dans le lait condensé, il faut ajouter de l'oxyde de zinc. Il faut ajouter $1 / 10^{\mathrm{e}}$ de l'acide lactique déterminé par dosage, pour compenser l'eau perdue au cours de la neutralisation.

Grossfeld [225] décrit une méthode permettant de déterminer l'indice d'acide de petites quantités de matières grasses. Il obtient des valeurs de 18,6 à 22,0 pour les matières grasses du beurre de lait de vache ; 0,8 à 6,4 pour les matières grasses du lait de femme. Le lait de femme ne contient done que très peu d'acide butyrique. Lucentini et Drago [226] donnent un nouvel indice pour les matières grasses du beurre, défini comme étant «le nombre de centimètres cubes d'une base décinomale permettant de neutraliser les acides solubles dans une solution saturée de sulfate de potassium ". Les matières grasses du beurre ont dans ces conditions un indice compris entre 18,6 et 20,9 ; le suif a un indice de 3 et le beurre de cacao, 5 .

\section{BIBLIOGRAPHIE}

[1] Davies (1936). Journal Dairy Res., 7, 75-96.

[2] TURner (1936). Bull. Univ. Mo. Agric. Exp. Sta., no 365, 30 pages.

[3] J ACOBSEN (1936). Journal Dairy Sci., 19, 171-76.

[4] Oxhey (1935). Journal Dairy Res., 6, 113-20.

[5] Csukas (1936). Chem. Abstr., 30, 1936.

[6] Hartley et Baker (1935). Journal Dairy Res., 6, 353-63.

[7] Stuffe (1936). Chem. Abstr., 30, 6461.

[8] Dahlberg, Kudera, Hening et Hucker (1936). Bull. N. Y St. Agr. Exp. Sta., $\mathrm{n}^{0} 239,16$ pages. 
[9] Susiura (1934). Journal Orient. Med., 30, 29-70 (Chem. Abstr., 1937, 31, 3163).

[10] Florentin (1937). Ann. Falsif., Paris, 30, 156-60.

[11] Rep. Minist. Hlth, Lond., 1934-35, 1935-36 and 1936-37.

[12] Sнан (1936). Indian Journal Vet. Sci., 6, 234-42.

[13] Pissarewsky (1936). Le Lait, 16, 465-84, 592-611, 711-23.

[14] GodDen et PUDDY (1935). Journal Dairy Res., 6, 307-12.

[15] Bergman et Turner (1937). Journal Dairy Sci., 20, 37-45.

[16] Bosworth et Helz (1936). Journal Biol. Chem., 112, 489-92.

[17] Helz et Bosworth (1935). Journal Biol. Chem., 116, 203-8.

[18] Green et Hilditch (1935), Biochem. Journal, 29, 1564-76.

[19] Hilditch et PAUL (1936). Biochem Journal, 30, 1905-14.

[20] Hilditoh (1937). Analyst, 62, 250-8.

[21] Vivario et Beneden (1936). Le Lait, 16, 113-18.

[22] Maynard, McKay et Madsen (1936). Journal Dairy Sci, 19, 49-53.

[23] Riemenschneider et Ellis (1936). Journal Biol. Chem., 113, 219-33.

[24] Choliet et Camus (1937). Le Lait, 17, 135-41.

[25] Gallup (1936). Industr. Engng Chem., Anal. ed. 8, 123-4.

[26] GoRyaev (1937). Milchw, Zbl., 66, 65-9.

[27] Richardson (1936). Journal Dairy Sci., 19, 749-52.

[28] Gallup, Kuhlman et Waldby (1937). Oil and Soap, 14, 124-6.

[29] Whetham et Hammond (1935). Journal Dairy Res., 6, 341-51.

[30] Dahlberg et Carpenter (1936). Journal Dairy Sci., 19, 541-51.

[31] Монг (1936). Molkereiztg, Hildesh., 50, 1454-5.

[32] Монв et Welcm (1936). Dtsch. Molkztg, 57, 1057-9.

[33] Roland, Sorensen et, Whitaker (1937). Journal Dairy Sci,, 20, 213-18.

[34] Sharp, Trout et Guthrie (1937). Milk Pl. Mon., 26 (5), 32-34, 44.

[35] Brown, Thurston et Dustman (1937). Journal Dairy Sci., 20, 133-45.

[36] Anderson (1936). Milk Dlr, 26, (1), 60-5.

[37] Thurston, Brown et Dustman (1936). Journal Dairy Sci., 19, 671-28,

[38] Ross (1937). Milk. Pl. Mon., 26, (4), 26-39; 26, (5), 40-4.

[39] Doan et Myers (1936). Milk Dlr, 26, (1), 76-87.

[40] WeBb et Hileman (1937), Journal Dairy Sci., 20, 47-57.

[41] Stebnitz et Sommer (1937). Journal Dairy Sci., 20, 181-96, 265-80.

[42] Greenbank (1936). Oil and Soap, 13, 140-1.

[43] Alcott et Mattinl (1936). Oil and Soap, 13, 98-100.

[44] Brown, Peterson et Görtner (1936). Journal Dairy Sci., 19, 81-92.

[45] Whitnah et Caulfield (1934). Rep. Kans agric. Exp. Sta., 1932-4, p. 83-5.

[46] Sommerfield (1935). Z. Vergl. Physiol., 22, 414-30.

[47] Theophilus, Hansen, Snyder Wood et Olmstead (1935). Bull. Idaho agric. Exp. Sta., no 212, p. 3-19.

[48] Sharp et McInerney (1936). Journal Dairy Sci., 19, 573-9.

[49] Groн et Altin (1936). Chem. Abstr., 30, 7589.

[50] Perov (1935). Chem. Abstr., 29, 6612.

[51] KopACZEWSKI (1936). Le Lait, 16, 801-10.

[52] Pederson (1936). Biochem. Journal, 30, 948-60, 961-70. 
[53] Lampitt et Bushill (1935). XVe Cong. Chem. Ind (Brussels), p. 982-7.

[54] Martin (1935). An. Soc. esp. Fis. Quim., 33, 905-30.

[55] RAY (1936). Le Lait, 36, 148-52.

[56] Todtenhaupt (1936). Z. Textilindustr., 39, 314-15.

[57] Косн (1936). Z. Textinlindustr., 39, 306-14.

[58] Fischer (1936). Nature, Lond., 137, 576-7.

[59] Rowland (1937). Journal Dairy Res., 8, 1-5, 6-15.

[60] Miller et Calvery (1936). Journal Biol. Chem., 116, 393-408.

[61] Perkins (1935). Bull. Ohio Agric. Exp. Sta., no 548, p. 62.

[62] Bhagrat (1936). Curr. Sci., 5, 297-98.

[63] Davies (1935). Journal Dairy Res, , 6, 363-8.

[64] Olson (1934). S. Dak. Agric. Exp. Sta. Rep., p. 30-1.

[65] BuRR et Witt (1935). Molkereiztg, Hildesh., 49, 1703-4.

[66] PYNe (1935). Sci. Proc. R. Dublin Soc., 21, 223-9.

[67] Ling (1936). Journal Dairy Res., 7, 145-55.

[68] Hermano et Clarayall (1935). Philipp. Journal Sci, 57, 323-8.

[69] Caulfield et Riddell (1935). Cornell Vet., 25, 333-43.

[70] Davies (1935, 1936), Rep. nat. Inst. Dairy, Reading.

[71] Blood et Rowlands (1936). Journal Dairy Res., 7, 47-54.

[72] Sharp et Struble (1935). Journal Dairy Sci., 18, 527-8.

[73] Leulier, Revol et Pascard (1937). C. R. Soc. Biol. Paris, 124, 1114-5.

[74] Waters et ZüRN (1935). Z. Untersuch. Lebensmitt., 70, 353-5.

[75] Müller (1936). Dtsch. Molkztg., 57, 1837-8.

[76] MaseK (1936). Le Lait, 16, 941-2.

[77] Jensen et Winther (1936). Milchw. Z bl., 65, 63-6.

[78] Ritter (1936). Schweiz. Milchztg., 62, 493-4.

[79] Rougichitch et Dumitresca (1936). Arch Dis. Childh., 11, 61-4.

[80] Reder (1935), Proc. Okla. Acad,, Sci, 15, 49-50.

[81] Kay, Mattick et Folley (1937). Analyst, 62, 259.

[82] KRUkovsky et SHARP (1936). Journal Dairy Sci. 19, 279-84.

[83] Sohloemer (1936). Milchw. Forsch, 17, 326-51 (Z. Untersuch. Lebensmitt, 71, 311-18).

[84] Kay et GRAham (1935). Journal Dairy Res, 6, 191-203.

[85] Kay et NeAve (1937). Dairy Ind., 2, 5-7.

[86] Anderson, Herschdörffer et Neave (1937). Analyst, 62, 86-95.

[87] Koppejan (1936). Chem. Weekbl., 33, 747.

[88] Basu et MuknerJee (1936). Journal Indian Chem. Soc. 13, 11-18.

[89] GangL et Liedi (1936). Mikrochem. Festschr. v. H. Molisch, p. 147-53. [90] Kasahara, Nosu, Kawamura et Fuji (1936). Jb. Kinderheilk, 147, 357-9.

[91] Krauss et Washburn (1936). J. Biol. Chem., 114, 247-52.

[92] Brock et Woolf (1936). Acta Brev. neerl. Physiol. 5, 80-1 (Chem. Abstr., 1937, 31, 3162).

[93] Ritter (1935-1936). Schweiz Milchztg, 61, 525, 531, 539; 62, 25, 331.

[94] J ACKson (1936). Journal Dairy Res., 7, 25-8.

[95] McDowall (1936). Analyst, 61 472-3.

[96] Polonovski (1936). Le Lait, 16, 232-4.

[97] KLUGE (1936). Z. Untersuch. Lebensmitt., 71, 232-45. 
[98] Burstein et Frum (1935). Z. Untersuch. Lebensmitt, 69, 421-31.

[99] Holwerda (1937). Chem. Abstr., 31, 165.

[100] Holm, Wright et Deysher (1936). Journal Dairy Sci., 19, 631-39.

[101] Horrall (1935). Bull. Ind, agric. Exp. Sta., n ${ }^{\circ} 401,31$ pages.

[102] Lampitt et Rooke (1936). Analyst, 61, 654-65.

[103] Reichard (1934), Z. anal. Chem. 99. 161-9.

[104] Mohargue (1934). Rep. Ky agric. Exp. Sta., p. 44-5.

[105] Trout et TAYLOR (1935). Quart. Bull. Mich. agric, Exp. Sta., no 18, p. $37-41$.

[106] Davies (1936). Agric. Progr,, 13. 112-15.

[107] Velu et Belle (1935). C. R. Soc. Biol. Paris, 119, 678-9.

[108] Kopaczewski (1936). Le Lait, 16, 356-9.

[109] Leviton et LeightoN (1936). Journal phys. Chem., 40, 71-80.

[110] Sirks (1936). Chem. Abstr., 30, 2649.

[111] Sharp, Myers et Guthrie (1936). Journal Dairy Sci., 19, 655-62.

[112] BaLdwin et Doan (1935-6). Journal Dairy Sci. 18, 629-38; 19, 225-33.

[113] Mantovani (1936). Chem. Abstr., 30, 173.

[114] Restivo (1935). Pediatria (Rev.), 43, 544-61.

[115] SrRks (1936). Exp. Sta. Rec., 74, 387-8.

[116] Rimpila et Palmer (1935). Journal Dairy Sci., 18, 827-39.

[117] Tracy et RaMSEY (1933-34). Rep. Ill. agric. Exp. Sta., p. 124-5.

[118] Lampitt et Bushili (1934). XIVe Congr. Chim. indust. (Paris), 6 pages.

[119] Tracy (1936). Milk Dlr, 25 (4), 30-32.

[120] Riddeli, Caulfield et Whitnah (1936). Journal Dairy Sci., 19, 157-64.

[121] Kugelmass (1937). Areh. Dis. Childh., 12, 25-44.

[122] CaULFiELd et Martin (1932-34). Rep. Kans, agric. Exp. Sta., p, 82-83.

[123] Palmer et Tarassuk (1936). Journal Dairy Sci, 19, 323-35.

[124] Lundstedt (1934). Milk Pl. Mon., 23 (7), 32-9.

[125] Charley (1936). Dairy Ind., 1, 19-20, 59-61.

[126] Davies (1937). Journal Soc. chem. Ind., Lond., 56, 10-13 T.

[127] Courtney (1936). Proc. 9th Ann. Sta. Coll. Wash. Inst, Dairying, p. 117-20.

[128] Caulfield et Riddell (1936). Journal Dairy Sci., 19, 235-242.

[129] VatLlant (1935). Le Lait, 15, 961-70.

[130] Mayorov (1935). Z. Untersuch Lebensmitt, 69. 301-5.

[131] Sohwarz et Fischer (1935). Milchw. Forsch, 17, 158-69.

[132] "Elact G. m. b. H. " (1936). D. R. P., 634-644, 1/9/36.

[133] Pien et Baisse (1936). Le Lait, 16, 921-41.

[134] Lyons (1936), Econ. Proc. R. Dublin Soc, 3, 19-38.

[135] Coulter et Coомвs (1936). Tech. Bull. Minn. agric. Exp. Sta., no 115, 39 pages.

[136] Richardison et Abbott (1936). Proc. 21 st. Ann. Meeting, West Div., Amer Dairy Sci. Assoc,, p. 63-9.

[137] Horn, MüHL et JuNGEMANN (1937). Landw, Jb, 84, 83-91.

[138] Platon, Humannson, Eden et Hansson (1935). Chem. Abstr., 29, 6964 .

[139] Hammer (1934). Proc. 7th Ann. Sta. Coll. Wash. Inst. Dairying, p. 16-20. 
[140] Hammer (1935). Journal Dairy Sci., 18, 579-81.

[141] Was et Csiszar (1936). Chem. Abstr., 30, 3107, 6838.

[142] Barnicoat (1935). Analyst, 60, 653-62.

[143] Kunze (1936). Microchemie, Festschr. v. H. Molisch, p. 279-89.

[144] Miohaelian et Hammer (1936). Res. Bull. Ia Agric. Exp. Sta., no 205, p. 303-14.

[145] Virtanen, Mansikkola et Tilka (1937), Chem. Abstr., 31, 1110.

[146] Ritter ot Christen (1935). Landw. Jb. Schweiz, 49, 749-60.

[147] Hammer, Stahly, Werkman et Miohaelian (1935). Res. Bull. Ia Agric. Exp. Sta., no 191. p. 381-407. (Proc. Iowa Acad. Sci., 42, 73-6.

[148] Virtanen et Tarnanen (1936). Acta. Chem. Fenn., 9 B, 2 (Chem. Abstr., 1936, 30, 3106).

[149] Barnidoat (1935). Journal Dairy Res., 6, 397-406.

[150] Ruene (1937). Nat. Butt. Cheese Journal, 28, (4), 20-2.

[151] Lemoigne et Monguillon (1935). Ann. Falsif., Paris, 28, 228-30.

[152] Riddet, Hodgson, Levy et Sears (1937). Bull. N. Z. Dep, sci. industr. Res., n० 52, p. 1-16.

[153] Ritter (1936). Schweiz. Milchztg, 62, 529, 538, 541, 543.

[154] Overman (1936). Nat, Butt. Cheese Journal, 27, (24), 6-7.

[155] Barnicoat (1936). Refrig. Engng, 32, 335-6.

[156] Hood (1936). Canad. Dairy Ice Cr. Journal, 15, (7), 45-49.

[157) Kieferle et Senss (1935). Dtsch. Molkztg., 56, 1115-18.

[158] Gilmour et ArUp (1935). Ice cold Stor., 38, 120.

[159] Guthrie, Scheib et Mark (1936). Journal Dairy Sci., 19, 267-78.

[160] Jacobsen (1936). Journal Dairy Sci., 19, 125-36.

[161] KNUDSEN (1935). Chem. Zbl. 2, 1104.

[162] Trambios et Pasztor (1935). Chem. Abstr., 29, 5937.

[163] French (1936). Bull. imp. Inst. Lond. 34, 32-4.

[164] Greene (1935). Food Industr. 7, 441-2.

[165] Powelu (1936). Journal Dairy Sci., 19, 305-311.

[166] Koestier et Petermann (1936). Landw. Jb. Schweiz., 53, 103-20.

[167] Dolby, MoDowall et MoDowell (1937). Journal Dairy Res., 8, 74-85, 86-91.

[168] MoDowall et Dolby (1936). Journal Dairy Res., 7, 156-75.

[169] Koestler (1936). Landw. Jb. Schweiz., 50, 976-1028.

[170] Sanders, Matheson et Burkey (1936). Journal Dairy Sci., 19, 395-404

[171] TUCKey (1936). Nat. Butter and Cheese J., 27, (23), 22-6.

[172] McDowall (1936). N. Z. J. Sci. Tech., 18, 137-364.

[173] SHERWOOD (1935). Journal Dairy Res., 6, 407-21.

[174] Grimmer et Lange (1936). Milchw, Forsch., 17, 352-75.

[175] Parist et Devito (1936). Atti Accad. Lincei, 23, 149-52.

[176] England et Mecham (1936). Bull. Md. Agric. Exp. Sta., no 393 , p. 569-93.

[177] BURGWALd et Mooney (1937) Milk Dlr, 26, (4), 40, 54; 5, (5), 72. [178] KenNy (1936). Milk Dlr, 25 (8), 78-84.

[179] Jackson, How et HoAR (1936). Journal Dairy Res. 7, 284-91. [180] LoveLESS (1935). Food, 5, 52-8.

[181] Stebnitz (1937). Ice Cr. Tr. J. 33, (3), 35-36. - Mack (1937). Ice Cr. Tr. J., 32 (11), 33-4. 
[182] Tracy (1936). Ice Cr. Tr. J., 32 (3), 31-2.

[183] Dahle (1936). Dairy Ind., 1, 147-9.

[184] Lucas et Jensen (1937). Quart. Bull. Mich. agric. Exp. Sta., no 19, p. 147-55.

[185] Young (1935). Proc. 8th Ann. Sta. Coll. Wash. Inst. Dairying, p. 35-45.

[186] Stebnitz et Sommer (1936). Journal Dairy Sci. 19, 101-15.

[187] Davies (1935). J. Soc. chem. Ind. Lond., 54, 338-41 T.

[188] Kraft Phoenix Cheese Corporation (1935). B. P. 434058, $26 / 8 / 35$.

[189] Mason et Hutcheson (1936). B. P. 447, 105, 13/5/36.

[190] Flanigan et Supplee (1935). U. S. P., 2,023,014, 3/12/34.

[191] Meade et Leckie (1936). Milk. Pl. Mon., 25, (8), 28, 29.

[192] TAussig (1935). Le Lait, 15, 1087-101.

[193] Pizer (1936). Chem. et Ind., 55, 704.

[194] MoDowall (1936). Dairy Res. Inst. (N. Z.) Publ., 79, 3 pp.

[195] British Standards Institution (1936). Publ. no 696, Pts land 11.

[196] Golding (1934). Agric, Progr., 11, 115-19.

[197] Koenig (1936). Z. Untersuch Lebensmitt., 71, 1-8.

[198] Leithe (1936). Z. Untersuch. Lebensmitt, 71, 245-53.

[199] Voorst (1936). Chem. Weekbl. 33, 5-8, 42-3.

[200] McDowall et MoDoweli (1936). Analyst, 61, 387-90.

[201] Leroy (1936). C. R. Acad. Agric. Franc., 22, 609-12.

[202] TURNAU (1937). Z. Untersuch Lebensmitt., 73, 26-30.

[203] Voorst (1935). Chem. Weekbl, 32, 478-9.

[204] Polonovski et Martin (1935-6). Chem. Abstr., 29, 6656; 30, 3534.

[205] SchWARz et Schlag (1936). Molkereiztg, Hildesh, 50, 2593-6.

[206] Tilici et Dragulescu (1936). Z. Untersuch. Lebensmitt, 72, 121-37.

[207] Mohler et Forter (1936). Mitt. Lebensm. Hyg., Bern, 27, 113-30.

[208] Schwarz, Fischer et Kahleit (1935). Milchw. Forsch., 17, 170-80.

[209] Maclachlan (1935). Analyst, 60, 752.

[210] Fouassier (1937). Ann. Falsif., Paris, 30, 94.

[211] Stoxle (1936). Analyst, 61, 336.

[212] TAPERNOUX (1936). Le Lait, 16, 832-40.

[213] Lefebure (1935). Journal Pharm. Belg., 17, 853, 921, 1057.

[214] WILle (1936). Michw. Forsch., 18, 111-15.

[215] Jones et Davies (1935). Biochem. J. 29, 978-81.

[216] Richardion (1936). Journal Ass. off. Agric. Anal., 19, 389-90.

[217] Ferguson et Racicot (1936). Journal Ass. off. Anal., 19, 476-80.

[218] Stuвbs (1935). Analyst, 60, 600-7, 607-10,

[219] Oestermann (1935). Pharm. Zentralh., 76, 300.1.

[220] Denis-Lester (1936). Bull. Dep. Agric. S. Afr., no 156, 14 pages.

[221] Elsdon et Stubis (1937). Analyst, 61, 382-6.

[222] Magraw et Copeland (1936). Cereal Chem., 13, 541-9.

[223] Munsey (1935). Journal Ass. off. Agric. Chem., 18, 573-7.

[224] Harrison (1935). Journal Ass. off. Agric, Chem., 18, 645-6.

[225] Grossfeld (1935). Z. Untersuch. Lebensmitt., 70, 459-70.

[226] Ludentini et Drago (1935). Ann. Chim. appl., Roma, 25, 388-91. 